\title{
Monte Carlo Simulation of Dember Effect in $n$-InAs under Subpicosecond Laser Pulse Excitation
}

\author{
V.L. MaLeVich* \\ Institute of Physics, National Academy of Sciences of Belarus \\ Kuprevich 1, bl. 2, 220141 Minsk, Republic of Belarus
}

\begin{abstract}
Monte Carlo method is used to simulate photo-Dember effect and generation of electromagnetic terahertz pulses in $n$-InAs excited by femtosecond laser radiation. Dynamics of electric field and transport of carriers were considered self-consistently. It is shown that, under excitation of semiconductor by laser pulses with photon energies $<1.1 \mathrm{eV}$, the Dember photovoltage reaches a peak value (it can be in tens times larger than the typical Dember photo-electromotive force at stationary illumination) through 50-100 fs after excitation and then fades while oscillating with plasma frequency. Excitation of semiconductor by radiation with a greater photon energy $(>1.1 \mathrm{eV})$ results in inter-valley transfer of photoelectrons and the photovoltage to decrease.
\end{abstract}

PACS numbers: 72.40.+w, 78.47.+p, 78.20.Bh

\section{Introduction}

Recently generation of terahertz $(\mathrm{THz})$ electromagnetic radiation from semiconductor surface excited by femtosecond laser pulses has been widely studied [1-4]. It has been established that this effect is caused by the pulse of photocurrent arising from ultra-fast redistribution of photoexcited carriers in semiconductor subsurface region. The photo-electromotive force (photo-emf) inducing the photocurrent results from the two mechanisms - barrier and diffusion. The relative contribution of these two mechanisms in the photo-emf depends on the laser photon energy, surface depletion field, and other parameters of a semiconductor [5]. For example, in $n$-GaAs the contribution of diffusion component in photo-emf is insignificant, and the barrier mechanism caused by the spatial redistribution of electrons and holes under the surface depletion field is dominant. However, for narrow-gap semiconductors, such as $n$-InAs and $n$-InSb, the main contribution

*e-mail: vitaly@optoinform.bas-net.by 
to the generation of $\mathrm{THz}$ pulses is due to the photo-Dember effect caused by the diffusion separation of photoexcited electrons and holes [4-7].

Under stationary excitation the typical value of Dember photo-emf is about $10^{-2} \mathrm{~V}[8,9]$. However, for $n$-InAs irradiated by the laser pulse at a wavelength of $800 \mathrm{~nm}$, photoexcited electrons for a time of about 1-2 ps after photoexcitation have a large kinetic energy and are capable to diffuse more effectively than the thermal carriers. In this case the diffusion photovoltage can reach the value far beyond the stationary photo-emf, and as a result the photo-Dember effect can be mainly responsible for THz-pulse generation in $n$-InAs [4-7].

\section{Results and discussion}

In the present work an ensemble Monte Carlo method [10, 11] is used to simulate photo-Dember effect and THz-pulse generation in $n$-InAs excited by $100 \mathrm{fs}$ laser pulses. The dynamics of electric field and carrier transport are considered selfconsistently using the macroparticles method (described in detail elsewhere [11]). The numerical simulations were carried out for the sample of $n$-InAs with ionised donor concentration of $1.8 \times 10^{16} \mathrm{~cm}^{-3}$ which was excited by 100 fs laser pulse with photon energy in the range of $0.4-1.55 \mathrm{eV}$. The semiconductor optical and band structure parameters, deformation potentials, and the energy of optical and inter-valley phonons were taken from $[12,13]$.
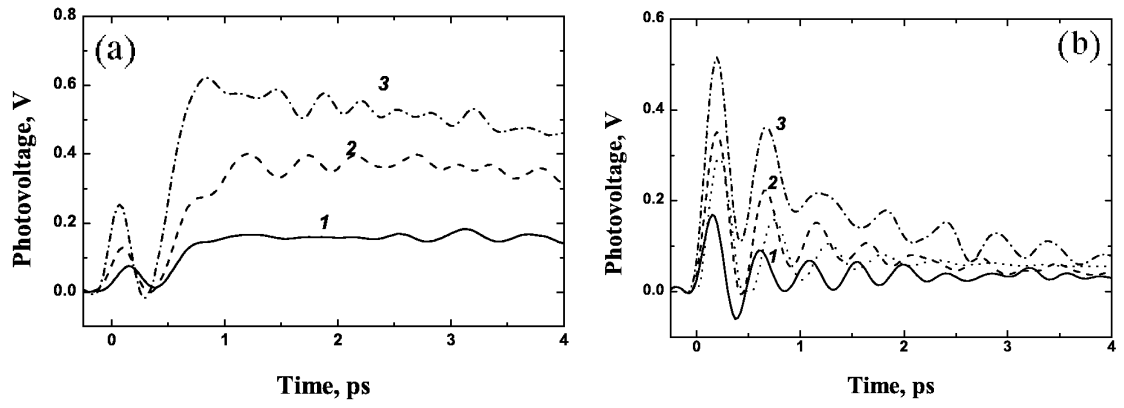

Fig. 1. Calculated temporal development of photovoltage in $n$-InAs excited by $100 \mathrm{fs}$ laser pulses (a) with-photon energy of $1.55 \mathrm{eV}$ and fluences $\left(\mu \mathrm{J} / \mathrm{cm}^{2}\right): 1-1 ; 2-2$; $3-5$. (b) The exciting pulses photon energies $(\mathrm{eV}): 1-0.4,2-0.8,3-1$; for all calculated curves the surface density of exciting photons is equal to $4 \times 10^{12} \mathrm{~cm}^{-2}$.

Calculated time dependences of the photovoltage arising in $n$-InAs excited by 100 fs laser pulse with a photon energy of $1.55 \mathrm{eV}$ are presented in Fig. 1a for three excitation fluences. As one can see, the photovoltage grows in time non-monotonically and achieves its peak value in a time of about 1 ps after excitation. Simulations show that photoexcited electrons in a time less than 100 fs pass from the central $\Gamma$-valley into the lateral $L$ - and $X$-valleys, where they are very fast cooled down by emitting optical phonons and then in a time of about 1 ps return 
to the $\Gamma$-valley. Since the mobilities of electrons in the lateral valleys are much lower than that in the central valley, the effective spatial separation of carriers does not begin at once but only through some picoseconds after excitation, when photogenerated electrons return to the $\Gamma$-valley. It follows from calculations that in the first some picoseconds after excitation the photovoltage can reach the value $(0.5 \mathrm{~V})$ that is far in excess of the stationary Dember photo-emf. The enhancement of the Dember photo-emf can be explained in terms of the hot photoelectrons diffusion.

Results of simulations of the photovoltage in $n$-InAs under excitation with $100 \mathrm{fs}$ laser pulses with photon energies in the range of $0.4-1 \mathrm{eV}$ are presented in Fig. 1b. For all curves the surface density of exciting photons is supposed to be constant and equal $4 \times 10^{12} \mathrm{~cm}^{-2}$ (at a wavelength of $800 \mathrm{~nm}$ it approximately corresponds to the fluence of $\left.1 \mu \mathrm{J} / \mathrm{cm}^{2}\right)$. As it follows from calculations, the scattering of photoelectrons in the lateral valleys does not occur in this case, and the temporal dynamics of photovoltage (Fig. 1b) is substantially different from that in the previous case (Fig. 1a): the photovoltage achieves the peak value through approximately $100 \mathrm{fs}$ after photoexcitation or at a stage of ballistic transport of photoelectrons. The mechanism of photo-emf origin in this case is rather of a jet nature and is caused by asymmetric scattering of photoelectrons via their reflection from the surface of a semiconductor [14]. Comparing the curves in Fig. 1b and the line 1 in Fig. 1a (the surface density of the electron-hole pairs generated is the same for all curves), it can be seen that with an increase in the exciting photon energy photo-emf grows in the range of $0.4-1.1 \mathrm{eV}$ and then having reached a maximum starts to decrease. Thus, it is possible to draw a conclusion that in $n$ InAs the contribution of one electron-hole pair in photo-emf grows with increasing photon energy down to the value of $1.1 \mathrm{eV}$ that is the threshold energy for the transition of photoelectrons to the lateral valleys. This result can be understood when taken into account that the increase in photoelectrons kinetic energy with the growth of photons energy results in more effective diffusion separation of carriers. If the exciting photons energy exceeds $1.1 \mathrm{eV}$, there is an intensive scattering of photoelectrons into the lateral valleys that results in reduction of the photovoltage and the delay effect described above.

The distinguishing feature of photo-Dember effect dynamics in $n$-InAs is that the photovoltage oscillates in time with the plasma frequency defined by equilibrium electrons. The contribution of photoexcited electrons in coherent plasma oscillations is essentially suppressed here since the hot photoelectrons are characterised by a greater effective mass and are scattered more intensively in comparison with equilibrium electrons.

The calculated time dependences of the electric field of THz-pulse generated in $n$-InAs under femtosecond irradiation are presented in Fig. 2a. The Fourier analysis shows that the spectrum width of THz-pulse grows with an increase of the photoexcitation level. Under irradiation of semiconductor by laser pulse with 

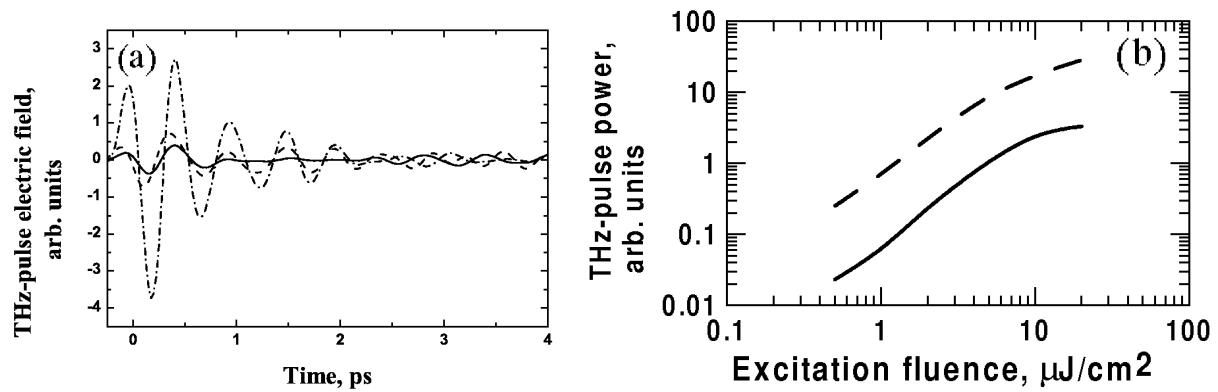

Fig. 2. (a) The electric field of THz-pulses generated from $n$-InAs under excitation by $100 \mathrm{fs}$ laser pulses with a photon energy of $1.55 \mathrm{eV}$ and at fluences of $1 \mu \mathrm{J} / \mathrm{cm}^{2}$ (solid line) and $2 \mu \mathrm{J} / \mathrm{cm}^{2}$ (dashed line); the dashed-dotted line corresponds to the excitation by pulse with $1 \mathrm{eV}$ photon energy and at a fluence of $1 \mu \mathrm{J} / \mathrm{cm}^{2}$. (b) Excitation fluence dependences of the intensity of THz-pulses generated from $n$-InAs excited by 100 fs laser pulses with photon energies of $1.55 \mathrm{eV}$ (solid line) and $1 \mathrm{eV}$ (dashed line).

photon energy $<1.1 \mathrm{eV}$ the spectrum width of THz-pulse is approximately 2 times narrower than that at the excitation with $1.55 \mathrm{eV}$ photons.

The calculated dependences on excitation fluence of intensities of $\mathrm{THz}$ radiation generated in $n$-InAs by femtosecond laser pulses are shown in Fig. 2b. The intensity of THz-pulse grows with an increase of the excitation fluence, and the efficiency of $\mathrm{THz}$ generation at $1 \mathrm{eV}$ excitation surpasses approximately on the one order the corresponding efficiency of generation at excitation with $1.55 \mathrm{eV}$ photon energy. It is significant that the substantial growth of THz-pulse generation was observed in $n$-InSb when the exciting radiation at $1560 \mathrm{~nm}$ wavelength was used instead of radiation at $800 \mathrm{~nm}[6]$.

\section{Summary}

In summary, Monte Carlo simulations of subpicosecond dynamics of photocarriers and electric field in $n$-InAs excited by femtosecond laser pulses show that the Dember photo-emf caused by the spatial separation of photoexcited carriers can reach comparatively a large value and be responsible for $\mathrm{THz}$-pulse generation.

This work was supported by ISTC, project B-1065.

\section{References}

[1] X.C. Zhang, B.B. Hu, J.T. Darrow, D.H. Auston, Appl. Phys. Lett. 56, 1011 (1990).

[2] H. Takahashi, H. Murakami, H. Ohtake, N. Sarukura, in: Solid-State Mid-Infrared Laser Sources, Eds. I.T Sorokina, K.L. Vodopyanov, in series Topics Appl. Phys., Vol. 89, Springer Verlag, Heidelberg 2003, p. 425.

[3] R. Kersting, J.N. Heyman, G. Strasser, K. Unterrainer, Phys. Rev. B 58, 4553 (1998).

[4] S. Kono, P. Gu, M. Tani, K. Sakai, Appl. Phys. B 71, 901 (2000). 
[5] M.B. Johnston, D.M. Whittaker, A. Corchia, A.G. Davies, E.H. Linfield, Phys. Rev. B 65, 165301 (2002).

[6] H. Takahashi, Y. Suzuki, M. Sakai, S. Ono, N. Sarukura, T. Sugiura, T. Hirosumi, M. Yoshida, Appl. Phys. Lett. 82, 2005 (2003).

[7] J.N. Heyman, P. Neocleous, D. Hebert, P.A. Crowell, T. Muller, K. Unterrainer, Phys. Rev. B 64, 085202 (2001).

[8] K. Seeger, Semiconductor Physics, Springer-Verlag, Wien 1973.

[9] A.V. Efanov, M.V. Entin, Fiz. Tekh. Poluprovodn. 20, 20 (1986) [Sov. Phys. Semicond. 20, 11 (1986)].

[10] R.W. Hockney, J.W. Eastwood, Computer Simulation Using Particles, McGraw-Hill, New York 1981.

[11] V.L. Malevich, Semicond. Sci. Technol. 17, 551 (2002).

[12] K. Brennan, K. Hess, Solid-State Electr. 27, 347 (1984).

[13] L.E. Vorobyev, Handbook Series on Semiconductor Parameters, Vol. 1, Eds. M. Levinshtein, S. Rumyantsev, M. Shur, World Scientific, London 1996.

[14] V.I. Belinicher, S.M. Ryvkin, Zh. Eksp. Teor. Fiz. 81, 353 (1981) [Sov. Phys.JETP 81, 353 (1981)]. 